Superlubricity and Wearless Sliding in Diamondlike Carbon Films

\author{
Ali Erdemir \\ Energy Technology Division \\ Argonne National Laboratory \\ Argonne, IL 60439
}

\begin{abstract}
The submitted manuscript has been created by the University of Chicago as Operator of
Argonne National Laboratory ("Argonne") under Contract No. W-31-109-ENG-38 with the

U.S. Department of Energy. The U.S. Government retains for itself, and others acting on its

behalf, a paid-up, nonexclusive, irrevocable worldwide license in said article to reproduce,

prepare derivative works, distribute copies to the public, and perform publicly and display
\end{abstract}

publicly, by or on behalf of the Government.

November 2001

Invited Paper presented at the 2001 MRS Fall Meeting,

November 26-30, 2001, Boston, MA

*Work supported by the U.S. Department of Energy, Office of Science, under Contract W-31-109-Eng-38. 


\title{
Superlubricity and Wearless Sliding in Diamondlike Carbon Films
}

\author{
Ali Erdemir \\ Energy Technology Division \\ Argonne National Laboratory \\ Argonne, IL 60439
}

\begin{abstract}
Diamondlike carbon (DLC) films have attracted great interest in recent years mainly because of their unusual optical, electrical, mechanical, and tribological properties. Such properties are currently being exploited for a wide range of engineering applications including magnetic hard disks, gears, sliding and roller bearings, scratch resistant glasses, biomedical implants, etc. Systematic studies on carbon-based materials in our laboratory have led to the development of a new class of amorphous DLC films that provide extremely low friction and wear coefficients of 0.001 to 0.005 and $10^{-11}$ to $10^{-10} \mathrm{~mm}^{3} / \mathrm{N} . \mathrm{m}$, respectively, when tested in inert-gas or high-vacuum environments. These films were produced in highly hydrogenated gas discharge plasmas by a plasma enhanced chemical vapor deposition process at room temperature. The carbon source gases used in the deposition of these films included methane, acetylene, and ethylene. Tribological studies in our laboratory have established a very close correlation between the composition of the plasmas and the friction and wear coefficients of the resultant DLC films. Specifically, the friction and wear coefficients of DLC films grown in plasmas with higher hydrogen-tocarbon ratios were much lower than films derived from source gases with lower hydrogento-carbon ratios. Fundamental tribological and surface analytical studies have led us to conclude that hydrogen (within the film, as well as on the sliding surfaces) is extremely
\end{abstract}


important for the superlubricity and wearless sliding behavior of these films. Based on these studies, a mechanistic model is proposed to explain the superlow friction and wear properties of the new DLC films.

Key Words: Diamondlike carbon, superlubricity, wearless sliding, friction and wear mechanisms.

\section{INTRODUCTION}

Tribologically, carbon-based materials and coatings (such as diamond, diamondlike carbon [DLC], graphite, graphite fluoride, glassy carbon, carbon-carbon or carbon-graphite composites) have been playing very important roles in combating or controlling friction and wear between sliding, rolling, or rotating tribo-components of a wide range of moving mechanical assemblies. Some of them (such as graphite, diamond, and DLC) can provide very low friction and wear coefficients when applied as thin films on tribological surfaces, while others (i.e., certain carboncarbon composites) can be tailored to attain very high friction and thus be used as brake materials [1,2]. Graphite has been available and used as a solid lubricant in numerous 
tribological applications for a long time [3]. Graphite fluoride is another carbon-based solid with excellent friction and wear properties [4]. Recently developed boron-containing carbon-carbon composites are proved to be extremely wear resistant and at the same time very lubricious over a wide temperature range (providing friction coefficients of 0.04-0.1 from room temperature to $\left.500^{\circ} \mathrm{C}\right)$ [5]. Under certain conditions, even bucky-balls $\left(\mathrm{C}_{60}\right)$ [6] and hollow carbon nanotubes [7] were shown to provide low friction when present on sliding tribological surfaces.

In general, friction is the result of a combination of chemical, physical, and mechanical interactions between two sliding surfaces; deformation, fracture, and third-body interactions can also play significant roles. Mechanical interlocking between two physically rough surfaces leads to high friction, as does too much chemical reaction or adhesive interaction (i.e., ionic, metallic, covalent, van der Waals, electrostatic, magnetic) between two sliding surfaces. The presence or absence of chemisorbed and/or physisorbed films (mainly by-products of test environment) may also significantly affect friction. Theoretically, one should expect superlow friction from two atomically smooth surfaces between which no adhesive and/or chemical interactions occur. Unfortunately, when brought into intimate contacts, most solid surfaces interact both chemically and physically, and when such surfaces are forced to slide over one another, such interactions lead to friction.

Among the many solids, carbon-based materials in general and diamond and DLC films in particular offer, perhaps, the best possibility for overcoming surface interactions and, hence, achieving superlow friction and wear on sliding contact interfaces. In fact, the systematic studies on carbon-based materials and coatings in our laboratory since the late 1980s have led to the discovery of amorphous carbon films that provide extremely low friction and wear coefficients 
[8-15]. One of the films that we optimized over the years provided friction and wear coefficients of 0.001 to 0.005 and $10^{-11}$ to $10^{-10} \mathrm{~mm}^{3} / \mathrm{N} . \mathrm{m}$, respectively, when tested in inert gases or high vacuum $[14,15,16]$. This film was synthesized in a highly hydrogenated gas discharge plasma that contained $75 \%$ hydrogen and $25 \%$ methane or $90 \%$ hydrogen and $10 \%$ acetylene. The results of numerous tribological tests in our laboratory and elsewhere have consistently demonstrated the existence of a very close correlation between the composition of the gas discharge plasmas and the friction and wear coefficients of the DLC films. Specifically, DLC films grown in plasmas with higher hydrogen-to-carbon ratios had much lower friction and wear coefficients than did films derived from source gases with lower hydrogen-to-carbon ratios. Fundamental tribological and surface analytical studies have led us to conclude that hydrogen (within the film, as well as on the sliding surfaces) is extremely important for the superlubricity and wearless sliding behavior of these films. Based on these findings, a mechanistic model is proposed to explain the superlow friction and wear properties of these DLC films. 


\section{MAJOR CAUSES OF FRICTION IN CARBON FILMS}

As mentioned earlier, two major causes of friction in carbon-based materials and coatings are physical roughness and chemical or adhesive interactions between their sliding surfaces. If rubbing surfaces are physically rough, extensive mechanical interlocking can occur between their surface asperities and thus lead to high friction. This situation is very typical of rough, polycrystalline diamond films with highly faceted diamond grains that protrude from the surface. Systematic studies of such surfaces by earlier researchers have established a correlation between surface roughness and friction coefficients [17-19]. In general, they found that the smoother the films, the lower the friction coefficients.

Apart from physical roughness, chemical and/or adhesive interactions between sliding carbon surfaces may also play major roles in friction. The adhesive interactions may mainly be the result of interatomic bonding (such as ionic, metallic, covalent, and hydrogen) and/or physical attractions (such as van der Waals, electrostatic, capillary, and magnetic forces). In the case of carbon-based materials in general and diamond and DLC films in particular, adhesive interactions may mainly be the result of covalent bonds, van der Waals forces, $\pi-\pi^{*}$ interactions, capillary forces, and electrostatic attractions. Ionic, metallic, or magnetic attractions do not exist in carbon coatings (unless they are doped with magnetic metals or compounds). Among the many bonds mentioned, covalent bonds are the strongest, and, if they exist between sliding carbon surfaces, can be the dominant cause of friction [20-23]. Covalent-bond interaction which can readily occur between sliding diamond surfaces at high temperatures, leads to very high friction [24]. In high vacuum, such surfaces also interact mostly by covalent bonding and experience high friction $[23,25,26]$. Van der Waals bonding, which exists between most surfaces brought into contact or very close proximity of each other, is one of the weakest and most 
desired kind of bonding between sliding surfaces, because very little lateral force is necessary to overcome it. The lamellar sheets of $\mathrm{MoS}_{2}$ and a few other layered solids are held together by van der Waals forces, and that is why such layered solids provide excellent lubricity under dry sliding conditions.

The presence or absence of chemisorbed and/or physisorbed films (mainly from environmental species) on sliding carbon surfaces may also have significant effects on friction. Among others, oxygen and water molecules have the greatest effect on friction of both hydrogenated and hydrogenfree DLC films. For example, in dry air and inert gases, friction coefficients of 0.001-0.05 appear feasible with hydrogenated DLC films $[8,12,14,15,27-30]$. However, in humid air, the friction coefficients may increase to more than 0.1 . In highly moist test environments, water molecules can easily precipitate on the sliding surfaces of carbon films and give rise to capillary forces that can add to friction.

In most sliding contacts, rubbing surfaces experience wear. Some of the wear debris particles may be trapped at the sliding contact interface and undergo physical grinding and chemical reaction with species in their surroundings. For example, in the case of steel rubbing against itself in open air, the debris particles consist mostly of iron oxides. The shear rheology of these oxides has a strong influence on the frictional properties of such sliding contact interfaces. These particles (sometimes referred to as "third bodies") may be smeared on one or both sliding surfaces as thin films (referred to as "transfer layers"), and the shear rheology of such films may also have strong influence on friction. In the case of diamond and DLC films rubbing against steel or ceramic counterfaces, the debris particles from the wear of carbon films have a 
disordered graphitic microstructure and are often smeared on the rubbing surfaces of steel and ceramic balls as thin transfer layers [9,10,31-35]. In most cases, formation of such transfer layers on sliding-ball surfaces is very beneficial and often leads to low friction. In these cases, the shear mainly occurs between original DLC films and the layers transferred onto rubbing-ball surfaces.

\section{DESIGN OF SUPERLUBRICIOUS DLC FILMS}

Based on the information provided above, one should theoretically expect superlow friction from an atomically smooth surface that is also chemically very inert and thus experiences no adhesive bonding or chemical interactions with the counterface material during sliding contacts. Among the many solids, carbon-based coatings in general and DLC in particluar offer perhaps the best possibility for meeting such conditions and hence providing superlow friction. DLC films are amorphous and hence very smooth. When deposited on atomically smooth or highly polished surfaces (such as silicon wafers or sapphire disks), they are not expected to alter or increase the original surface roughness of substrate materials. By controlling deposition parameters and source gas chemistry, one can make DLC films that are chemically very inert and at the same time very hard and rigid. Extreme surface smoothness is needed to achieve the least amount of asperity-asperity interaction, whereas high chemical inertness is necessary to prevent adhesive interactions. Furthermore, the high mechanical hardness and rigidity of DLC films ensure the smallest contact area by which most physical and chemical interactions occur. The main question is "how can we prepare films with such extreme qualities?" In the following paragraphs, details of the inception, preparation, and tribological demonstration of such superlow-friction carbon films will be presented.

At present, most thin solid films are produced by physical- and chemical-vapor-deposition (PVD 
and CVD) methods. To produce DLC films, a plasma-enhanced-chemical-vapor deposition (PECVD) is favored (because of its simplicity and cost-effectiveness). These films can also be produced by ion-beam-assisted deposition, magnetron sputtering, arc physical vapor deposition, laser ablation, and a few other methods. The superlow-friction DLC films presented in this study were produced in a PECVD system at room temperature; they were 1-1.5 $\mu \mathrm{m}$ thick and deposited on the highly polished surfaces of silicon wafers, sapphire, and AISI M50 and H13 steel substrates. The carbon source gases used were pure methane, acetylene, ethylene, and ethane. We have also obtained hydrogen-free DLC films from outside sources for comparison. The source gases were blended with $\mathrm{H}_{2}$ up to $90 \%$ to attain a highly hydrogenated gas discharge plasma. As will be elaborated later, the use of extra hydrogen was extremely important for the synthesis of superlow friction carbon films presented here. Specifically, systematic tribological studies in our laboratory over the years have revealed a close correlation between film properties and the hydrogen-to-carbon $(\mathrm{H} / \mathrm{C})$ ratio in the gas discharge plasmas. This ratio is essentially zero for films produced from a solid carbon target in an arc PVD system, whereas it is 1 for $\mathrm{C}_{2} \mathrm{H}_{2}, 2$ for ethylene, and 4 for $\mathrm{CH}_{4}$. To obtain $\mathrm{H} / \mathrm{C}$ ratios of 10 or more, we added hydrogen into the gas discharge plasmas.

The PECVD of DLC involved, first, the sputter-cleaning of the substrate material in an argon gas discharge plasma for $30 \mathrm{~min}$. The RF-bias voltage applied to these substrates was $1200-1700 \mathrm{~V}$. The next step was to produce a thin (50-70-nm-thick) silicon bond layer which was applied by sputtering silicon from a silicon target. In some cases, silane $\left(\mathrm{SiH}_{4}\right)$ gas was also used to form the bond layer on the surface. For synthesis of DLC films, the carbon-bearing source gases (such as $\mathrm{CH}_{4}$ and $\mathrm{C}_{2} \mathrm{H}_{2}$ ) and hydrogen gas were bled into the chamber until a gas pressure of 1013 mtorr is attained. The final step was to deposit the DLC films by introducing $\mathrm{CH}_{4}$. The RF 
bias applied to the specimen holder was 400-600 V. The use of RF bias prevents charge buildup during film deposition (in as much as DLC films are generally insulating). Figure 1 shows the surface morphology and cross-sectional microstructure of a typical DLC film produced by the method described above.

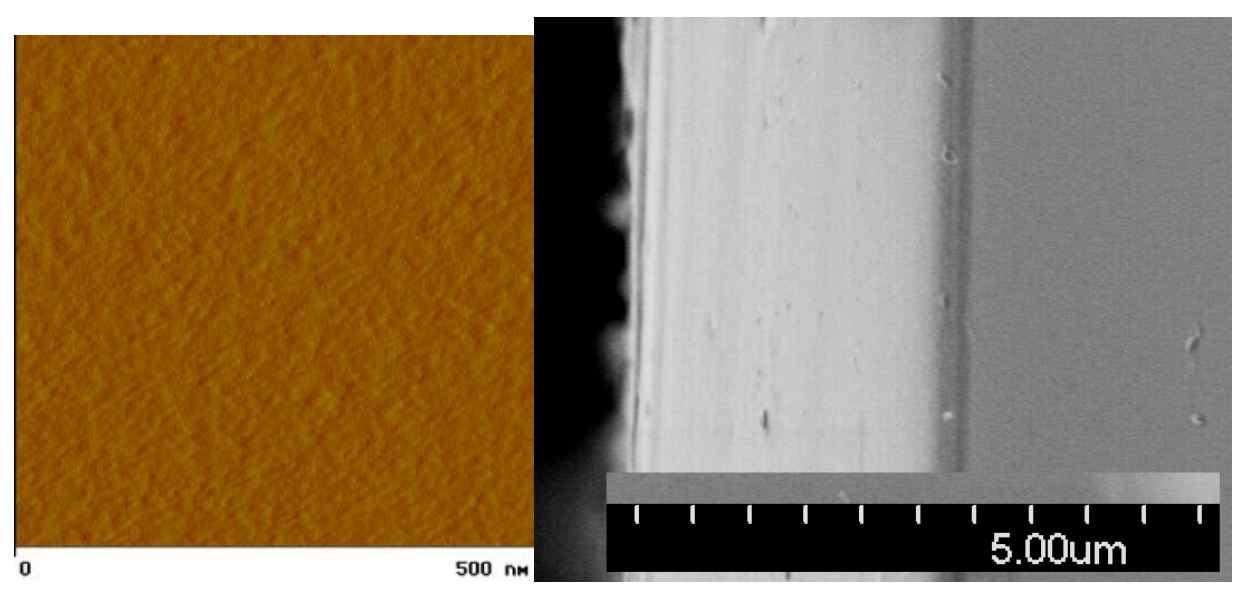

(a)

(b)

Figure 1. (a) Surface atomic force microscopy (AFM) and (b) cross-sectional scanning electron microscopy (SEM) morphology of a DLC film produced by PECVD on silicon wafer.

\section{TRIBOLOGICAL CHARACTERIZATION OF SUPERLOW FRICTION CARBON}

\section{FILMS}

The tribological characterization of DLC films can easily be accomplished with a ball-on-disk tribometer. For the present case, the tribological tests were performed in a dry nitrogen environment under contact loads of 5-10 N at sliding velocities of $0.2-0.5 \mathrm{~m} / \mathrm{s}$. The sliding 
distance was $2-5 \mathrm{~km}$. To measure the true friction coefficient of the DLC coating, the coated balls were rubbed against the coated disks. The Vickers hardness of the substrates and balls varied between $\approx 8$ and $35 \mathrm{GPa}$ (for steel and sapphire, respectively) and their surface roughness was better than $0.05 \mu \mathrm{m}$ centerline average (CLA). Most tests were run in dry nitrogen. The test chamber was thoroughly purged with dry $\mathrm{N}_{2}$ for at least $2 \mathrm{~h}$ after $0 \%$ relative humidity was shown on a hygrometer display unit. A few additional tests were run in open air with $50 \%$ relative humidity and in high vacuum (low $10^{-7}$ torr). The contact load used in the vacuum tribometer was $2 \mathrm{~N}$ and the sliding velocity was $1 \mathrm{~cm} / \mathrm{s}$.

The results of extensive tribological tests have confirmed that the chemistry of source gases (from which the DLC films are derived) has a dramatic effect on the friction and wear performance of DLC films. As shown in Fig. 2, the higher the amount of hydrogen in gas discharge plasmas, the lower the friction coefficients. A hydrogen free DLC film provided friction coefficients of 0.6 - 0.7 whereas a DLC film derived from a pure methane source exhibited friction coefficients of 0.015 0.02 [16]. Th $\mathrm{H} / \mathrm{C}$ ratio for methane source gas is 4 , whereas it is zero for the hydrogen-free DLC that was produced by a cathodic arc PVD process. Films derived from $\mathrm{C}_{2} \mathrm{H}_{2}$ (whose $\mathrm{H} / \mathrm{C}$ ratio is 1) provided friction coefficients of 0.3-0.4 under the same sliding conditions. The situation for ethylene, with a $\mathrm{H} / \mathrm{C}=2$, fit well into the general trend, as shown in Fig. 2. The actual friction traces of three of the films in Fig. 2 are presented in Fig. 3. Again, films grown in hydrogen-poor plasmas exhibit high and unsteady friction, whereas film grown in a methane plasma provide very steady and low friction. 


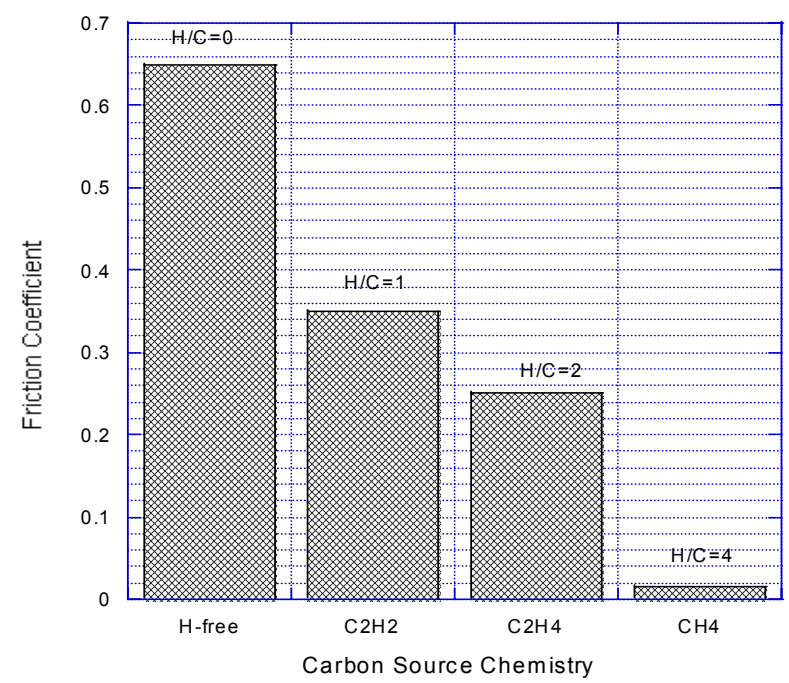

Figure 2. Friction coefficients of films derived from solid and gaseous carbon sources.

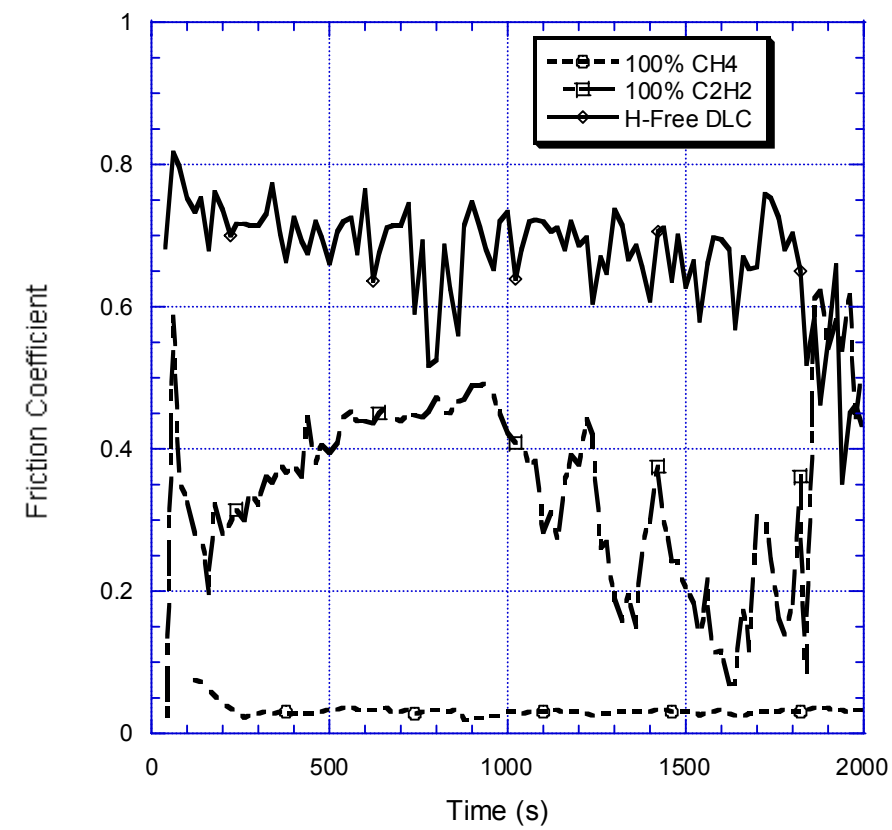

Figure 3. Actual frictional traces of films derived from $\mathrm{CH}_{4}, \mathrm{C}_{2} \mathrm{H}_{2}$ and pure carbon.

Addition of hydrogen (up to $90 \%$ ) to $\mathrm{C}_{2} \mathrm{H}_{2}, \mathrm{C}_{2} \mathrm{H}_{4}$, and $\mathrm{CH}_{4}$ source gases produced DLC films that 
were superlubricious. Figures 4-6 show the friction and wear performance of DLC films grown in gas discharge plasmas that ranged in composition from pure source gases (i.e., $\mathrm{C}_{2} \mathrm{H}_{2}, \mathrm{C}_{2} \mathrm{H}_{4}, \mathrm{CH}_{4}$ ) to highly hydrogenated plasmas. Wear rates are also included in these diagrams. These figures reveal a very close correlation between the friction and wear coefficients of DLC films and the $\mathrm{H} / \mathrm{C}$ ratios of the source gas plasmas used during deposition, regardless of the starting source gas. In general, the higher the $\mathrm{H} / \mathrm{C}$ ratio of the source gas, the lower the friction coefficient (suggesting that hydrogen plays an important role in the frictional behavior of DLC films). The lowest friction coefficient was achieved on sapphire substrates. Figure 7 shows the friction coefficient of a highly optimized DLC film produced on a pair of sapphire ball and disk. For comparison, the friction coefficient of an uncoated sapphire test pair is also included.

With respect to the wear of the superlubicious DLC films, the results in Figs. 4-6 demonstrate that wear rates decrease precipitously with increasing hydrogen in the source gas plasmas. In one case, the sliding test was interrupted at sliding distances of $10,50,150,250,500,5000$, and 50,000 m, and the surfaces of balls and disks were inspected by a noncontact three dimensional surface profilometer. Up to $50 \mathrm{~m}$, we could not detect any measurable wear scar on sliding-ball surfaces. At $150 \mathrm{~m}$, a minor wear scar had formed but its size remained fairly constant up to $5000 \mathrm{~m}$. At the 50,000-m mark, the wear scar was much larger and the underlying steel ball was exposed, while the coating on the disk side was still intact. In another lifetime test, a 1.5- $\mu \mathrm{m}$-thick DLC film on a steel substrate lasted $>17$ millions cycles (that translates into a 1300-km sliding distance). Again, the film on the ball side was worn out, but the film was still present on the disk side. 


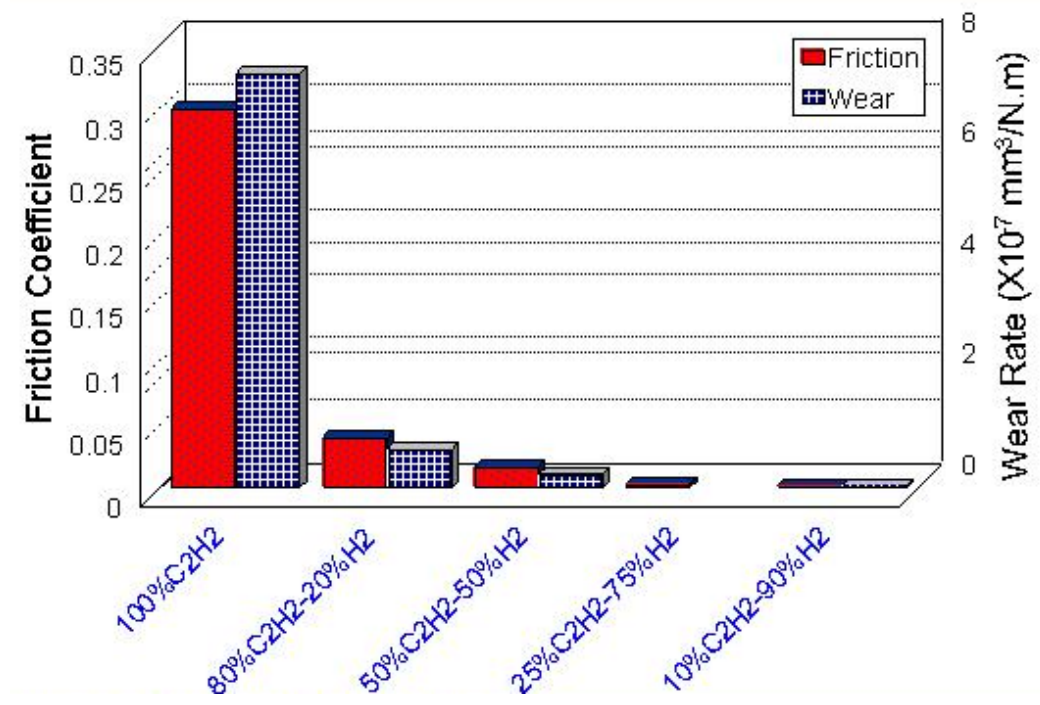

Figure 4. Effect of source gas chemistry on friction coefficients of DLC films derived from $\mathrm{C}_{2} \mathrm{H}_{2}$ (Note that $\mathrm{H} / \mathrm{C}$ ratio is 1 for $100 \% \mathrm{C}_{2} \mathrm{H}_{2}$ whereas, for $10 \% \mathrm{C}_{2} \mathrm{H}_{2}+90 \% \mathrm{H}_{2}$-containing plasma, it is 10).

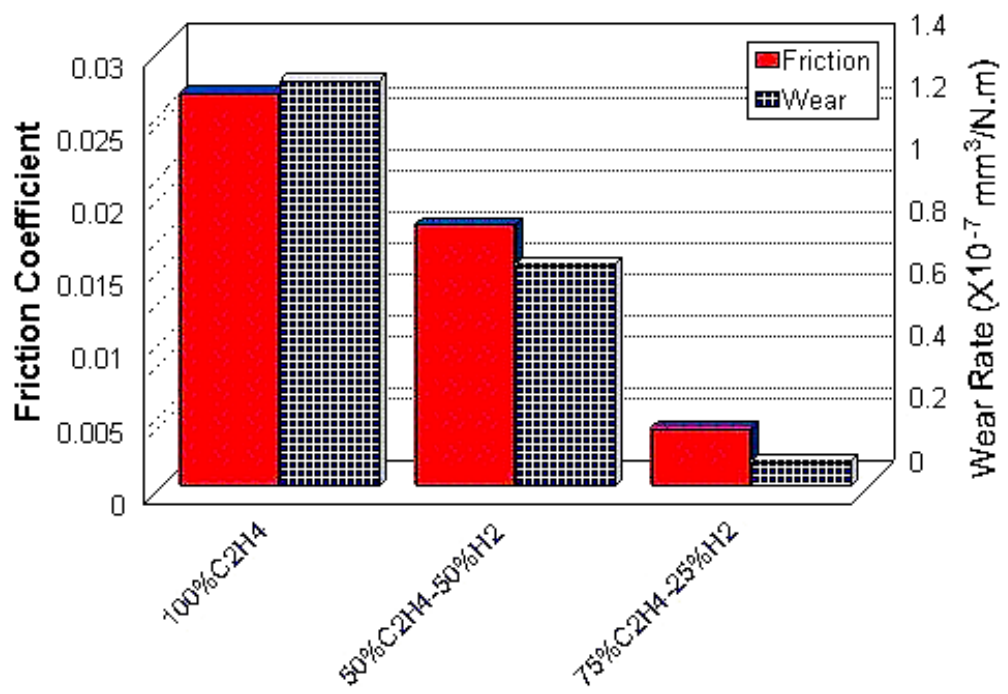

Figure 5. Effect of source gas chemistry on friction coefficients of DLC films derived from $\mathrm{C}_{2} \mathrm{H}_{4}$ (Note that $\mathrm{H} / \mathrm{C}$ ratio is 2 for $100 \% \mathrm{C}_{2} \mathrm{H}_{4}$, wheras it is 5 for $25 \% \mathrm{C}_{2} \mathrm{H}_{4}+75 \% \mathrm{H}_{2}$-containing plasma). 


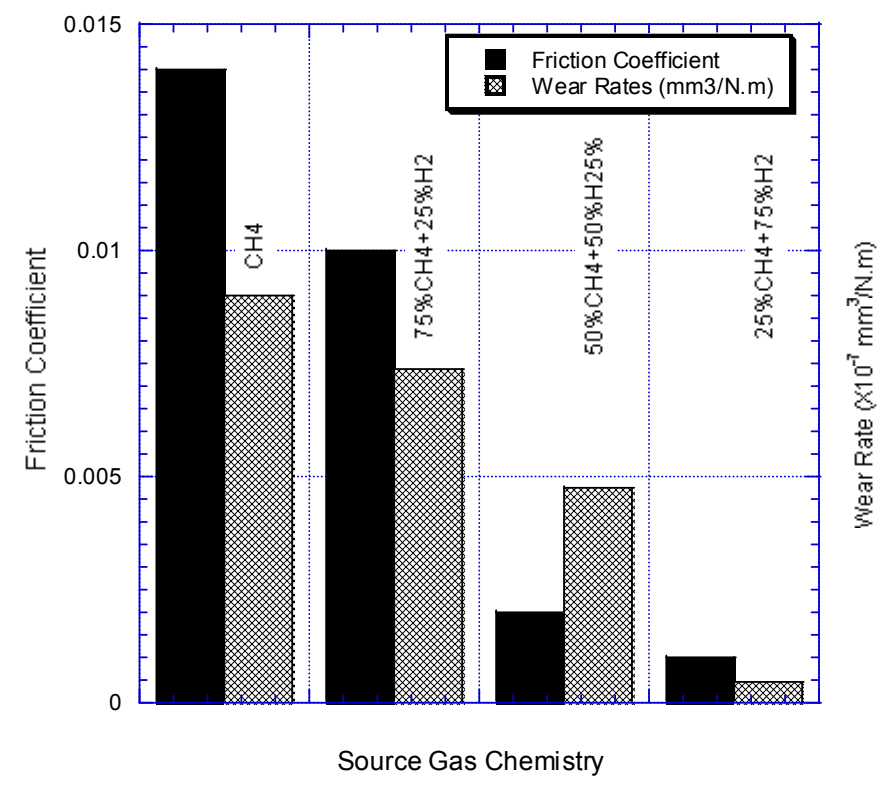

Figure 6. Effect of source gas chemistry on friction and wear coefficients of DLC films derived from $\mathrm{CH}_{4}$. 


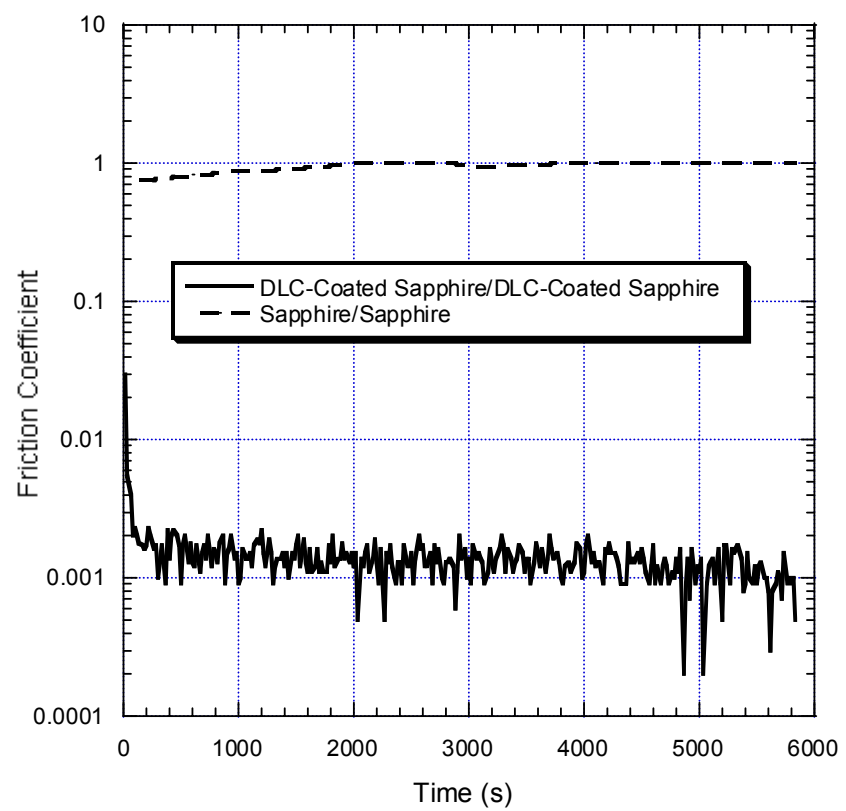

Figure 7. Frictional performance of sapphire when sliding against itself and of highly optimized DLC film sliding against itself in dry nitrogen.

\section{MECHANISM OF SUPERLUBRICITY OF CARBON FILMS}

The friction results in Figs. 2-6 suggest that hydrogen in gas discharge plasmas (from which the DLC films are derived) has a strong influence on the frictional performance of DLC films. Films grown in hydrogen-free or -poor plasmas exhibit high friction, whereas those grown in highly hydrogenated plasmas provide low friction. In general, the higher the amount of hydrogen in the plasma, the lower the friction coefficients of the resultant DLC films. The lowest friction coefficients, i.e., $0.002-0.005$, were provided by films grown in plasmas that contained $75-90 \%$ hydrogen. Beyond these values, it became very difficult to grow films.

Based on the results presented above, the following mechanistic explanation is provided for the superlubricious nature of our DLC films. It is well known that the affinity of hydrogen toward 
carbon is chemically very strong. Specifically, hydrogen bonds strongly to carbon and effectively passivates its un-occupied or free $\Phi$-bonds. In bulk diamonds or thin diamond films, surface carbon atoms are mostly passivated by hydrogen and/or other environmental species, such as oxygen, hydroxyl ions, etc. Once passivated, such surface carbon atoms become chemically very inert, causing very little adhesive interactions with counterface materials, thereby providing low friction [21-25]. This explanation is widely accepted for the low-friction behavior of diamonds. $\mathrm{C}-\mathrm{H}$ bonding is covalent and extremely strong (stronger than single $\mathrm{C}-\mathrm{C}$ bonds); hence, its removal from surface is very difficult. It can be removed from diamond surfaces at very high temperatures. When sliding tests are performed in high vacuum, hydrogen can be removed by mechanical means, and if the exposed $\Phi$-bonds are not repassivated, the covalent bond interactions can occur and lead to high friction.

When extra hydrogen is used during DLC deposition, several important events occur and determine the structural chemistry of the DLC films presented in this paper. First, it can lead to increased hydrogen concentration within the bulk, as well as on the surface. Most of these hydrogen atoms are paired with $\Phi$-bonds, but some unbonded free hydrogen may also exist as interstitials. The presence of molecular hydrogen within the films is also feasible. A high concentration of hydrogen within the DLC films and on the surface should effectively diminish or even eliminate the possibility of residual unoccupied $\Phi$-bonds that could participate in adhesive interactions during sliding. Free hydrogen within the films serves as a reservoir and can replenish or replace those hydrogen atoms that may have been lost due because of thermal heating and/or mechanical action during sliding. 
Second, hydrogen is highly effective in etching out or removing $\mathrm{sp}^{2}$-bonded- or graphitic-carbon precursors during deposition. The removal of such graphitic carbons prevents the formation of planar graphitic clusters that can give rise to B-B* interactions. When DLC films are prepared in highly hydrogenated gas discharge plasmas, strong $\mathrm{C}-\mathrm{H}$ bonding, rather than $\mathrm{C}=\mathrm{C}$ double bonding, should be favored. As explained above, the existence of residual B-bonding which can result from $\mathrm{C}=\mathrm{C}$ double bonds in $\mathrm{DLC}$, can give rise to friction. Finally, some of the carbon atoms (at least those on the surface) could be dihydrated, i.e., two hydrogen atoms were bonded to each carbon atom on the surface; this can occur on the unreconstructed (100) surfaces of diamond structures under some special or supercritical conditions that may have been created by energetic hydrogen bombardment in a highly hydrogenated discharge plasma. The existence of dihydrated carbon atoms on the surfaces will increase the hydrogen density of these surfaces and provide better shielding or passivation and thus superlow friction. Such a friction model is presented in Fig. 8 for partially dihydrated sliding DLC surfaces.

As mentioned earlier, other forces such as van der Waals and capillary forces and electrostatic attraction/repulsion, may also be present and may cause adhesion and hence friction at the sliding interfaces of DLC films. Because friction tests were run in a clean, dry nitrogen environment, the extent of capillary forces due to moisture precipitation on the sliding surfaces should be minimal or essentially absent. The van der Waals forces will be present at the sliding interfaces, but their relative contributions to overall frictional force should be insignificant because we used very high contact loads in our tests. Because the DLC films are, in general, dielectric, their sliding surfaces can certainly accumulate static electrical charge; hence the main question is whether these charges will cause attraction or repulsion. When the free electrons of 
hydrogen atoms pair with the dangling $\Phi$-bonds of carbon atoms, the electrical charge density is permanently shifted to the other side of the nucleus of the hydrogen atom and away from the surface. Such a shift in charge density allows the positively charged hydrogen proton in its nucleus to be closer to the surface than the electron that is used up by the $\Phi$-bond of the surface carbon atoms. Therefore, the creation of such a dipole configuration at the sliding interface should give rise to repulsion rather than attraction between the hydrogen-terminated sliding surfaces of the DLC films.

\section{Load}

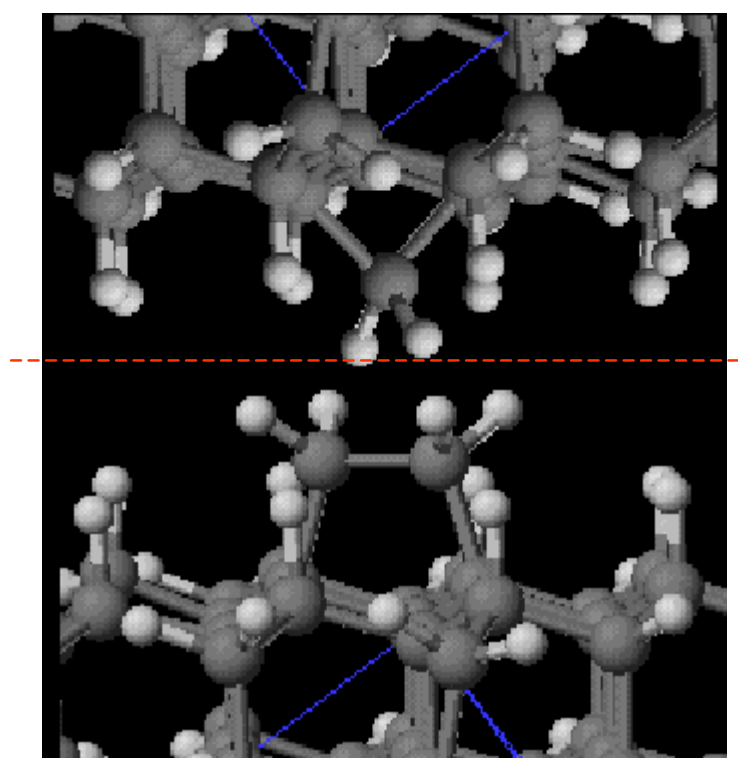

Partially di-hydrated Sliding carbon surfaces

\section{Load}
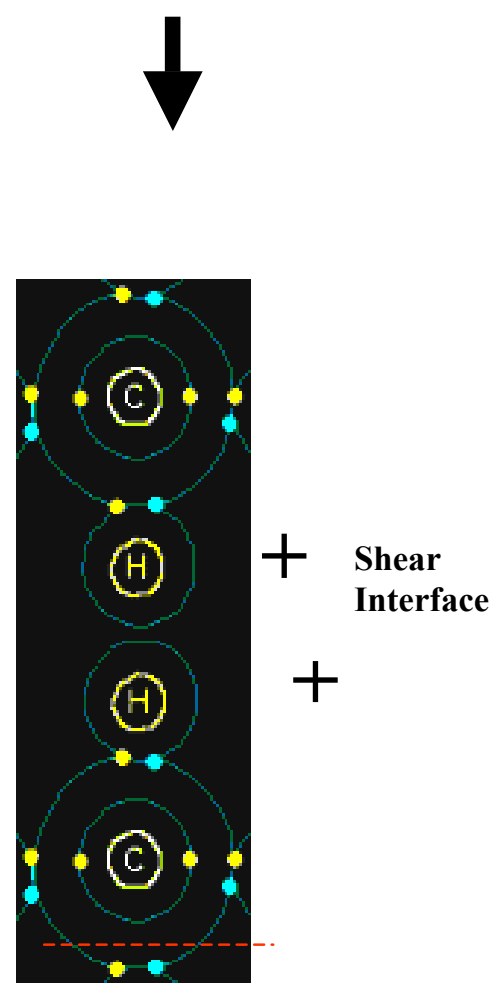

H-terminated

C atoms 
Figure 8. Mechanistic model for superlow friction behavior of (a) hydrogenated DLC surfaces with partial dihydration and (b) individual carbon atoms terminated by hydrogen.

The wear resistance of the DLC films correlated well with their friction performance. Specifically, films with higher friction coefficients suffer higher wear rates, whereas those with low or superlow friction suffer very little wear, as shown in Figs. 4-6.

\section{SUMMARY AND FUTURE DIRECTION}

Unlike most other coatings, DLC films enjoy a combination of low friction and high wear resistance under a wide range of sliding contact conditions; hence, they offer unique possibilities for a wide range of engineering applications. The results presented here demonstrate that the friction and wear properties of these films can further be improved by controlling the source gas chemistry. Specifically, when source gases are blended with additional hydrogen, the resultant films become superlubricious. Mechanistically, superlubricity and wearless sliding are attributed to a fully hydrogen-terminated carbon surface that does not interact chemically or physically with the sliding counterfaces. Using the principles presented here, one can design carbon-based two- and three-dimensional microstructures and/or machines with unique tribological properties.

\section{ACKNOWLEDGMENTS}

This work is supported by the U.S. Department of Energy, Office of Science, under Contract W-31- 
109-Eng-38. The author thanks his students and collaborators who participated in the preparation, testing, and characterization of DLC-coatings presented in this paper.

\section{REFERENCES}

1. A. Erdemir and C. Donnet, in Modern Tribology Handbook, edited by B. Bhushan, (CRC

Press, Roca Raton, FL, 2000), p. 871.

2. A. Erdemir, ibid., p. 787.

3. R. H. Savage, J. Appl. Phys., 19, 1 (1948).

4. R. L. Fusaro and H. E. Sliney, ASLE Trans., 13, 56 (1970).

5. A. Erdemir, G. Fenske, and D. Busch, United States Patent \# 6,255,234: Ultralow friction carbon composites for extreme temperature applications, 2001.

6. B. Bhushan, B. K. Gupta, G. W. Van Cleef, C. Capp, and J. V. Coe, Appl. Phys. Lett., 62, 3253 (1993).

7. M. R. Falvo, R. M. Taylor, A. Helser, V. Chi, F. P. Brooks, S. Washburn, and R. Superfine, Nature, 397, 236 (1999).

8. A. Erdemir, M. Switala, R. Wei, and P. Wilbur, Surf. Coat. Technol., 50, 17(1991).

9. A. Erdemir, F. A. Nichols, X. Z. Pan, R. Wei, and P. Wilbur, Diam. Rel. Mater., 3, 119 (1993).

10. A. Erdemir, C. Bindal, G. R. Fenske, P. Wilbur, Tribol. Trans., 39735 (1996).

11. A. Erdemir, G. R. Fenske, A. Krauss, D. Gruen, T. McCauley, and R. Csencsits, Surf. Coat. Technol., 121, 565 (1999).

12. A. Erdemir, I. B. Nilufer, O. L. Eryilmaz, M. Beschliesser, and G. R. Fenske, Surf. Coat. Technol., 121, 589 (1999).

13. D. A. Ersoy, M. J. McNallan, Y. Gogotsi, and A. Erdemir, Tribol. Trans., 43, 809 (2000). 
14. A. Erdemir, O. L. Eryilmaz, I. B. Nilufer, and G. R. Fenske, Diam. Rel. Mater., 9, 632 (2000).

15. A. Erdemir, O. L. Eryilmaz, and G. Fenske, J. Vac. Sci. Technol., A18 1987 (2000).

16. A. Erdemir, Proc. the 44th Annual Meeting of the Society of Vacuum Coaters, Philadelphia, PA, SVC Publications, Albuquerque, NM 2001, p. 397.

17. I. P. Hayward, I. L. Singer, and L. E. Seitzman, Wear, 157215 (1992).

18. B. K. Gupta, A. Malshe, B. Bhushan, and V. V. Subramaniam, J. Tribol., 116, 445 (1994).

19. A. Erdemir, M. Halter, G. R. Fenske, A. Krauss, D. M. Gruen, S. M. Pimenov, and V. I. Konov, Surf. Coat. Technol., 94-96, 537 (1997).

20. M. N. Gardos and B. L. Soriano, J. Mater. Res., 5, 2599 (1990).

21. F. P. Bowden and J. E. Young, Proc. Roy. Soc. London, A208, 444-455 (1951).

22. D. Tabor: Adhesion and Friction, in The Properties of Diamond, ed. J. E. Field, (Academic Press, 1979), p. 325.

23. K. Miyoshi, J. Appl. Phys., 74, 4446 (1993).

24. M. N. Gardos, Surf. Coat. Technol., 113, 183 (1999).

25. D. Dugger, E. Peebles, and L. E. Pope, (1992), in Surface Science Investigations in Tribology, Experimental Approaches, Edited by Y.-W. Chung, A. M. Homolo, and G. B. Street, ACS Symposium Series: 485, (American Chemical Society, Washington D.C, 1992), pp. 72-102.

26. S. Chandrasekar, and B. Bhushan, Wear, 153, 79-89 (1992).

27. C. Donnet and A. Grill, Surf. Coat. Technol., 94/95 456 (1997).

28. J. A. Heimberg, K. J. Wahl, I. L. Singer, and A. Erdemir, Appl. Phys. Lett., 78, 2449 (2001).

29. H. Ronkainen, J. Koskinen, J. Likonen, S. Varjus, and J. Vihersalo, Diam. Rel. Mater. 3, 1329 (1993).

30. K. Miyoshi, J. J. Pouch, and S. A. Alterovitz, Mater. Sci. Forum, 52/53, 645(1989). 
31. Y. Liu, A. Erdemir, and E. I. Meletis, Surf. Coat. Technol. 82, 48 (1996).

32. Y. Liu, A. Erdemir, and E. I. Meletis, Surf. Coat. Technol., 94-95, 463 (1997).

33. D. S. Kim, T. E. Fischer, and B. Gallois, Surf. Coat. Technol., 49, 537(1991).

34. J. E. Olsen, T. E. Fischer, and B. Gallois, Wear, $200233(1996)$.

35. C. Donnet, M. Belin, J. C. Martin, J. M. Martin, A. Grill, and V. Patel, Surf. Coat. Technol., 68-69, 626 (1994). 